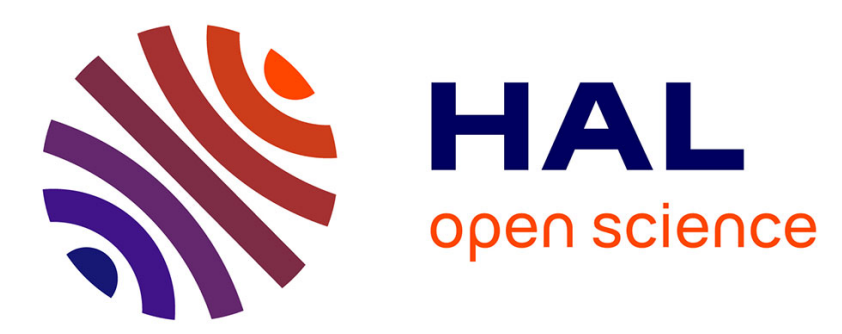

\title{
Structure cristallographique de multicouches métalliques et magnétiques étudiées par spectroscopie d'absorption $\mathrm{X}$
}

S. Pizzini, F. Baudelet, A. Fontaine, D. Chandesris, Hélène Magnan, A. Fert, F. Petroff, C. Marlière

\section{To cite this version:}

S. Pizzini, F. Baudelet, A. Fontaine, D. Chandesris, Hélène Magnan, et al.. Structure cristallographique de multicouches métalliques et magnétiques étudiées par spectroscopie d'absorption X. Journal de Physique IV Proceedings, 1992, 02 (C3), pp.C3-185-C3-189. 10.1051/jp4:1992326 . jpa00251531

\section{HAL Id: jpa-00251531 \\ https://hal.science/jpa-00251531}

Submitted on 1 Jan 1992

HAL is a multi-disciplinary open access archive for the deposit and dissemination of scientific research documents, whether they are published or not. The documents may come from teaching and research institutions in France or abroad, or from public or private research centers.
L'archive ouverte pluridisciplinaire HAL, est destinée au dépôt et à la diffusion de documents scientifiques de niveau recherche, publiés ou non, émanant des établissements d'enseignement et de recherche français ou étrangers, des laboratoires publics ou privés. 


\title{
Structure cristallographique de multicouches métalliques et magnétiques étudiées par spectroscopie d'absorption $\mathbf{X}$
}

\author{
S. PIZZINI, F. BAUDELET, A. FONTAINE, D. CHANDESRIS, H. MAGNAN, A. FERT', \\ F. PETROFF* et C. MARLIËRE** \\ LURE, Bat. 209D, Université Paris-Sud, 91405 Orsay, France \\ -Laboratoire de Physique des Solides, Universite Paris-Sud, 91405 Orsay, France \\ "* Laboratoire d'Optique, Universite Paris-Sud, 91405 Orsay, France
}

\begin{abstract}
The results of X-ray Absorption Spectroscopy (XAS) measurements on magnetic $\mathrm{Co} / \mathrm{Cu}$ and $\mathrm{Fe} / \mathrm{Cu}$ multilayers show the capability of this technique to point out the dependence of crystallographic phase on the layers thickness and the presence of structural anisotropies and strain in these multilayers.
\end{abstract}

\section{Introduction}

Les multicouches et les super-réseaux métalliques présentent des propriétés structurales et magnétiques inhabituelles. L'aimantation perpendiculaire observée récemment dans ces nouveaux matériaux, et la magnétoresistance géante, mise en évidence pour la première fois dans les multicouches $\mathrm{Fe} / \mathrm{Cr}$ sont les propriétés fondamentales de ces multicouches métalliques qui sont appelées à devenir des matériaux de base de l' enregistrement magnétique du futur $[1 ; 2]$.

Dans ces matériaux des structures cristallographiques inhabituelles, différentes de celles de l'état massif, peuvent être stabilisées. La sélectivité de la spectroscopie d'absorption de rayons $X$ (XAS) rend cette technique puissante pour charactériser la structure locale des multicouches [3]. Dans ce papier nous présentons les résultats de nos récentes mesures sur des multicouches $\mathrm{Fe} / \mathrm{Cu}$ et $\mathrm{Co} / \mathrm{Cu}$, où le couplage entre couches magnétiques montre des oscillations en fonction de l'épaisseur des couches non magnétiques [2].

\section{Méthodes éxpérimentales}

Les multicouches $\mathrm{Fe} / \mathrm{Cu}\left(\mathrm{tFe}_{\mathrm{e}}=15 \AA, \mathrm{t}_{\mathrm{Cu}}=6,13,24,42\right.$ and $\left.60 \AA\right)$ ont été preparées par pulvérisation dans une enceinte ultra-vide [4]. 
Les multicouches $\mathrm{Co} / \mathrm{Cu}$, préparées par évaporation thermique en ultra-vide sont déposées sur une couche tampon d'or, épaisse de $250 \AA$, elle-même déposée sur du verre.

La diffraction des rayons $X$ et les résultats de $R M N$ du cobalt [5] prouvent que les interfaces $\mathrm{Co} / \mathrm{Cu}$ sont très abruptes (rugosité rms $<4 \AA$ ). Les couches $\mathrm{Co}_{10} \mathrm{Cu}_{10}, \mathrm{Cog}_{5} \mathrm{Cu}_{5}$, $\mathrm{Co}_{5} \mathrm{Cu}_{3}, \mathrm{Co}_{3} \mathrm{Cu}_{5}, \mathrm{Co}_{3} \mathrm{Cu}_{3}$ (où sont portés en indice les nombres de monocouches de $\mathrm{Co}$ et $\mathrm{Cu}$ ) sont fortement texturées avec l'axe [111] perpendiculaire à la surface des échantillons.

Les mesures de XAS ont été realisées sur la station expérimentale D42 de LUREDCI equipée d'un monochromateur type "channel cut" utilisant la reflexion $311 \mathrm{du}$ silicium. Puisque les couches sont minces et déposées sur substrat, la détection des électrons secondaires émis à la suite de la création du trou profond est la méthode la plus efficace de mesure du spectre. Deux mesures avec les configurations géométriques classiques ont été réalisées: la direction de polarisation des photons $\mathrm{X}$ est parallèle ou quasi-perpendiculaire à la surface de l'échantillon. Les spectres EXAFS expérimentaux on été simulés en utilisant l'approximation en ondes planes et les phases et amplitudes expérimentales obtenues à partir de spectres de composés standards. Dans ce cas, phase et amplitude contiennent la correction "onde courbe" qui reste quasi-invariante lors que les distances interatomiques varient peu, ce qui est le cas.

\section{Resultats}

La figure 1 montre les transformés de Fourier (TF) des spectres EXAFS (Cu K) des multicouches $\mathrm{Cu} / \mathrm{Co}$, avec la polarisation des rayons $\mathrm{X}$ dans le plan des couches. Pour chaque multicouche, les TF (qui sont des quasi functions de distribution radiale) sont très proches de celle du spectre de $\mathrm{Cu}$ massif: la structure cubique faces centrées (cfc) des couches de $\mathrm{Cu}$ de ces Echantillons est très ordonné. La modélisation des spectres EXAFS (Tableau 1) montre en outre que les facteurs de Debye-Waller pour les couches de $\mathrm{Cu}$ sont pratiquement identiques à ceux du Cu massif.

Les distances des premiers voisins $\mathrm{Co}-\mathrm{Co}$ et $\mathrm{Cu}-\mathrm{Cu}$ dépendent de l'épaisseur des couches (Table 1). Les distances entre atomes dans le plan des couches (II) sont en général différentes des distances entre atomes dans des plans atomiques differents ( ), donnant une structure anisotrope. Dans le plan des couches, la distance Co-Co (2.52 $\AA$ ) est plus longue que celle du Co massif $(2.51 \AA)$, alors que la distance $\mathrm{Cu}-\mathrm{Cu}(2.54 \AA)$ est plus courte que celle du $\mathrm{Cu}$ massif $(2.556 \AA)$. Cette variation structurale est la réponse du réseau aux contraintes imposées par l'épitaxie de deux matériaux présentant un désaccord de maille à l'état massif. La tendance est naturellement de dilater le réseau du Co $\left(\mathrm{d}_{\mathrm{Co}}<\mathrm{d}_{\mathrm{Cu}}\right)$ et de contracter celui du Cu. La valeur moyenne des distances (II) dans ces multicouches est celle des matériaux massifs.

En revanche, dans la direction perpendiculaire aux plans, les distances des premiers voisins ne changent pas: elles sont celles des matériaux massifs. Les facteurs de Debye 
Waller (Tableau 1) montrent que les couches de Co sont beaucoup plus désordonnées que les couches de $\mathrm{Cu}$. Alors que le cuivre croît avec la structure cfc du massif, le cobalt adopte la structure métastable (cfc) imposée par le cuivre. Ce résultat important est aussi suggéré par la RMN du cobalt. Les fortes valeurs des facteurs de Debye-Waller sont dues probablement à la présence de défauts qui tendent à rétablir localement la structure hexagonale du Co massif.

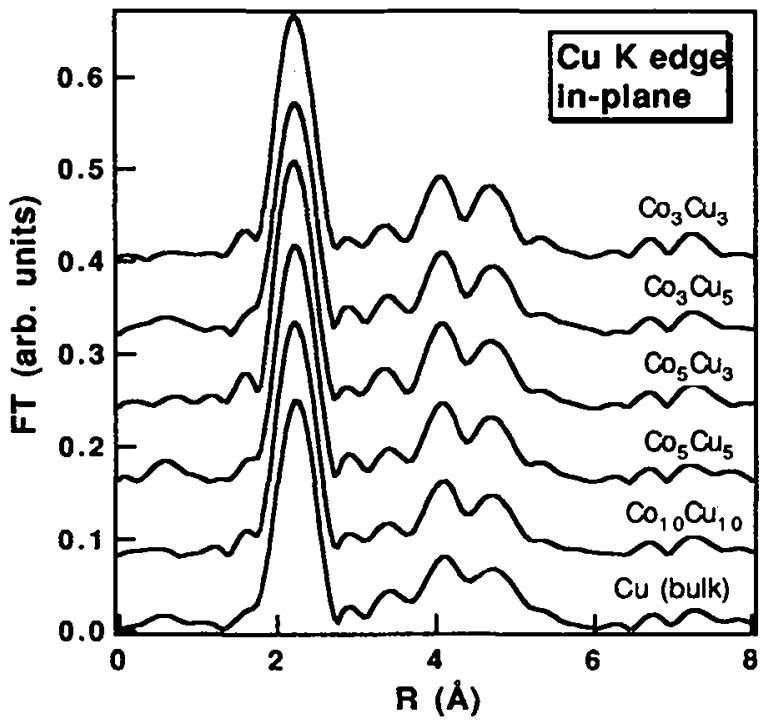

Figure 1: Les transformees de Fourier des spectres EXAFS des multicouches $\mathrm{Co}_{\mathrm{x}} \mathrm{Cu}_{\mathrm{y}}$ ( $x$ et y étant les nombres de monocouches de $\mathrm{Co}$ et de $\mathrm{Cu}$ ) au seuil $\mathrm{Cu} \mathrm{K}$, mesurées avec la polarisation des rayons $\mathrm{X}$ dans le plan des couches, sont comparés à la TF du spectre du Cu massif.

Table 1: Résultats des modélisations des spectres EXAFS aux seuils $\mathrm{Cu} \mathrm{K}$ et $\mathrm{Co} \mathrm{K}$, mesurés pour les multicouches $\mathrm{Cox}_{\mathrm{x}} \mathrm{Cu}_{\mathrm{y}}$, avec la polarisation du faiseau $\mathrm{X}$ dans le plan (in-plane) ou perpendiculaire au plan des couches (out-of-plane). R1 est la distance du premier voisin (Cu-Cu ou Co-Co). DW1 est le facteur de Debye-Waller, relatif à celui de $\mathrm{Cu}$ ou Co massifs.

\begin{tabular}{|c|c|c|c|c|}
\hline SAMPLE & $\begin{array}{c}\mathrm{R}_{1}(\mathrm{~A}) \\
\text { (in-plane) }\end{array}$ & $\begin{array}{l}\mathrm{DW}_{1}\left(\mathrm{~A}^{2}\right) \\
\text { (in-plane) }\end{array}$ & $\begin{array}{c}\mathrm{R}_{1}(\mathrm{~A}) \\
\text { (out-of-plane) }\end{array}$ & $\begin{array}{c}\mathrm{DW}_{1}\left(\AA^{2}\right) \\
\text { (out-of-plane) }\end{array}$ \\
\hline & 2556 & Cu K edge & & \\
\hline $\mathrm{Cu}$ (bulk) & 2.556 & - & 2.556 & - \\
\hline $\mathrm{Co}_{10} \mathrm{Cu} 10$ & 2.54 & 0.0001 & 2.55 & 0.0004 \\
\hline $\mathrm{Co}_{5} \mathrm{Cu} 5$ & 2.54 & 0.0000 & 2.55 & 0.0005 \\
\hline $\mathrm{Co}_{3} \mathrm{Cu}_{3}$ & 2.54 & -0.0005 & 2.55 & 0.0000 \\
\hline & & CoK edge & & \\
\hline Co (bulk) & 2.51 & & 2.50 & - \\
\hline $\mathrm{Co}_{10} \mathrm{Cu}_{10}$ & 2.52 & 0.0003 & 2.50 & 0.0008 \\
\hline $\mathrm{Co}_{5} \mathrm{Cu} 5$ & 2.52 & 0.0018 & 2.51 & 0.0019 \\
\hline$\left(\mathrm{Co}_{3} \mathrm{Cu}_{3}\right.$ & 2.52 & 0.0023 & 2.51 & 0.0023 \\
\hline
\end{tabular}



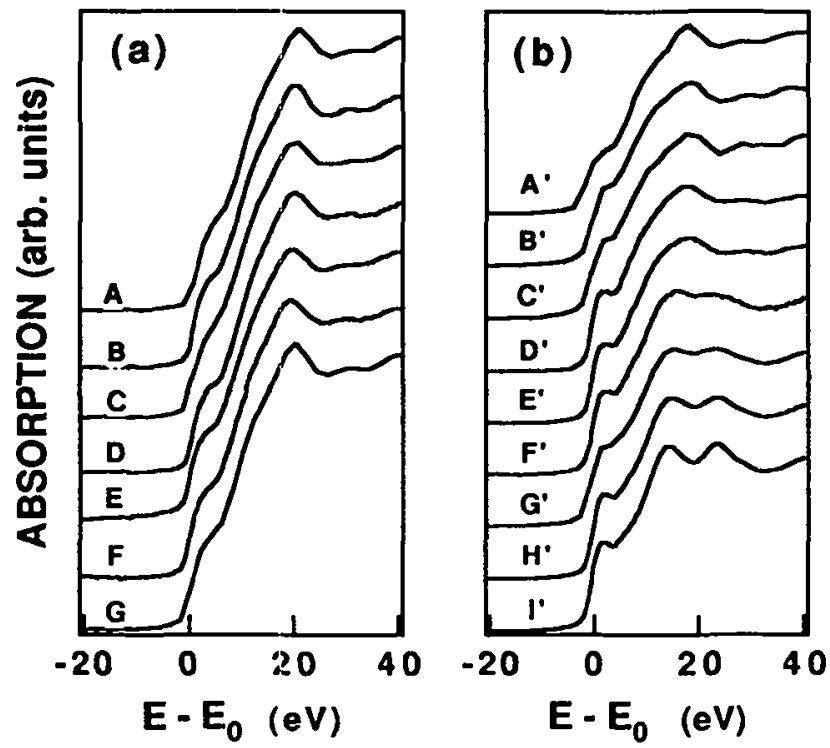

Figure 2: Seuils d'absorption $\mathrm{Fe} \mathrm{K}$ (a) et $\mathrm{Cu} \mathrm{K}$ (b) pour les multicouches (Fe $15 \AA$ / $\left.\mathrm{Cu}{ }^{\mathrm{C}} \mathrm{Cu}\right)\left(\mathrm{E}_{0}=8982 \mathrm{eV}\right)$ mesures avec la polarisation des rayons $X$ dans les plans ou

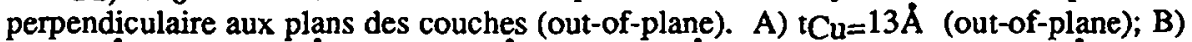
$\mathrm{tCu}^{\mathrm{a}}=13 \AA ; \mathrm{C}$ ) $\left.\mathrm{tCu}=24 \AA ; \mathrm{D}\right) \mathrm{tCu}=42 \AA ; \mathrm{E}$ ) $\mathrm{tCu}=60 \AA$ (out-of-plane) ; F) $\mathrm{tCu}=60 \AA ; \mathrm{G}$ ) $\mathrm{Fe}$

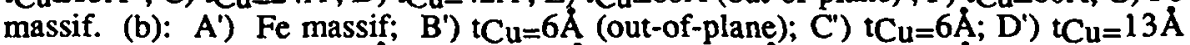

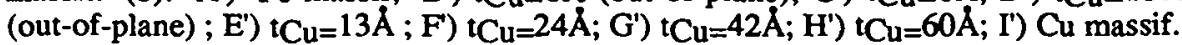

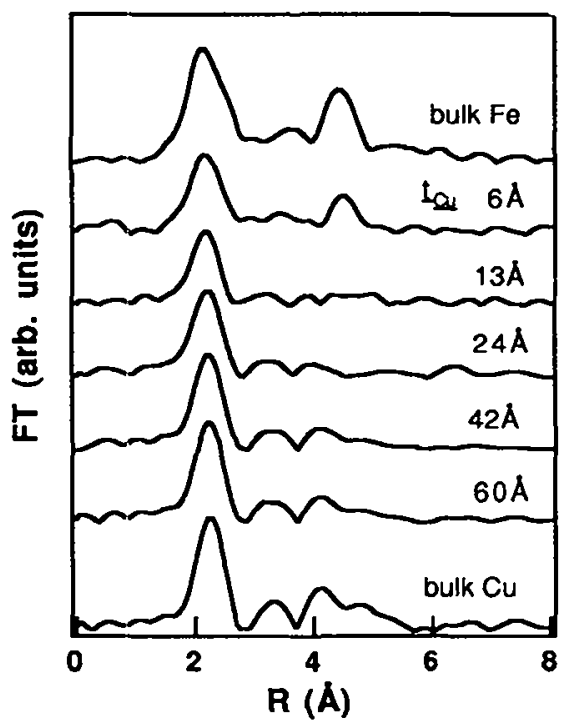

Figure 3: Les transformées de Fourier des spectres EXAFS au seuil $\mathrm{Cu} \mathrm{K}$ mesurées pour les multicouches $\left(\mathrm{Fe} 15 \AA / \mathrm{Cu} t_{\mathrm{Cu}}\right.$ ) avec la polarisation des rayons $\mathrm{X}$ dans le plan des couches sont comparées aux TF des spectres de Cu massif (cfc) et Fe massif (cc). 
La figure 2 montre les seuils d'absorption $\mathrm{Fe} \mathrm{K}$ et $\mathrm{Cu} \mathrm{K}$ pour les multicouches $\mathrm{Fe} / \mathrm{Cu}\left(\mathrm{t}_{\mathrm{Fe}}=21 \AA, \mathrm{t}_{\mathrm{Cu}}=6,13,24,42,60 \AA\right)$ comparés aux seuils de $\mathrm{Cu}$ et Fe massifs [7]. Le est cubique centré (cc), quelle que soit l'épaisseur du $\mathrm{Cu}$, alors que la structure des couches de $\mathrm{Cu}$ dépend fortement de leurs épaisseurs.

$\mathrm{La}$ forme des spectres d'absorption $\mathrm{Cu} \mathrm{K}$ change avec l'épaisseur évoluant de celle typique d'une structure cc pour $\mathrm{t}_{\mathrm{C} u}=6 \AA$, à celle d'une structure cfc pour $\mathrm{t}_{\mathrm{Cu}}=60 \AA$. La même tendance est montrée par les TF des spectres EXAFS au seuil $\mathrm{Cu} K$ (fig. 3). Les TF pour $t_{C u}=6 \AA$ montrent à grande distance les pics typiques d'une structure cc. Pour $t_{C u}=24,42$ et $60 \AA$ les TF montrent les distances typiques d'une structure cfc. La présence de pics bien définis à grande distance est l'indication d'une structure ordonnée. D'autre part la modélisation des spectres EXAFS montrent que le désordre dans ces couches de $\mathrm{Cu}$ est beaucoup plus important que dans le $\mathrm{Cu}$ massif. Les couches de $\mathrm{Cu}$ de $13 \AA$ se trouvent près du point de transition entre la structure cc et la structure cfc. Elles sont très désordonnées, comme l'indique l'absence de pics à grande distance dans les TF.

Les résultats de la modélisation des spectres d'EXAFS [7] montrent que pour les couches de cuivre plus fines ( $6 \AA$ et $13 \AA$ ) la distance du premier voisin $\mathrm{Cu}-\mathrm{Cu}$ (2.50$2.52 \AA)$ est courte et typique d'une structure cc $(2.556 \AA$ pour cfc). Pour $13 \AA \mathrm{de} \mathrm{Cu}$, la structure cc est très désordonnée. Pour des épaisseurs plus importantes, la structure relaxe vers la phase cfc tdu cuivre massif. Pour ces épaisseurs $(24,42$ et $60 \AA)$ la distance $\mathrm{Cu}-\mathrm{Cu}$ est plus longue, et typique du $\mathrm{Cu}$ cfc. Le processus de relaxation est lent, et les couches de $\mathrm{Cu}$ deviennent plus ordonnées au fur et à mesure que leurs épaisseurs augmentent.

\section{References}

[1] L.M. Falicoff, D.T. Pierce, S.D. Bader, R. Gronsky, K.B. Hathaway, H.J. Hopster, D.N. Lampeth, S.S.P. Parkin, G. Prinz, M. Salamon, I.K. Schuller, R.H. Victoria, J. Mater. Res. Vol. 5, No. 6, June 1990, et références

[2] F. Petroff, A. Barthélemy, D.H. Mosca, D.K. Lottis, A. Fert, P.A. Schroeder, W.P. Pratt, R. Loloee, S. Lequien, Phys. Rev. B 44 (1991) 5355

[3] F. Baudelet, Thèse de Doctorat, Université d'Orsay, 1991

[4] J.M. Slaughter, W.P. Pratt, P.A. Schroeder, Rev. Sci. Instrum. 60 (1989) 127

[5] S. Pizzini, F. Baudelet, A. Fontaine, M. Galtier, D. Renard, C. Marlière, soumis à Phys. Rev. B

[6] D.C. Koningsberger, R. Prins, X-ray Absorption. Principles, Applications, Techniques of EXAFS, SEXAFS and XANES, J. Wiley, 1988

[7] S. Pizzini, F. Baudelet, D. Chandersis, A. Fontaine, H. Magnan, J.M. George, F. Petroff, A. Barthélemy, A. Fert, R. Loloee, P.A. Schroeder, Phys. Rev. B (Rapid Communications) 46 (1992) 1253 